\title{
NLO properties and spectroscopic characterization of Y-shaped polymer using quantum chemical approach
}

\author{
Mahadevappa Naganathappa ${ }^{1}$, Ajay Chaudhari ${ }^{2}$, Balakrishna Kolli ${ }^{3}$, and Sampath Ravula ${ }^{4}$ \\ ${ }^{1}$ GITAM (Deemed to be University) Hyderabad Campus \\ ${ }^{2}$ The Institute of Science \\ ${ }^{3}$ GITAM University Hyderabad campus \\ ${ }^{4}$ GITAM Deemed to be University Hyderabad campus
}

May 13, 2020

\begin{abstract}
The present study reports nonlinear optical properties such as first and second hyper polarizabilities $(\beta$ and $\gamma)$ of Y-shaped polymer (P1) and substituted polymers. The basic Y-shaped polymer $(\mathrm{R}=\mathrm{R} 1=\mathrm{H})$ named as $\mathrm{P} 1$. Upon substitution of one OCH3 group in ortho position of Oxygen becomes polymer P2 (R1=H, R=OCH3) and two OCH3 group as P3 (R1=R=OCH3). We have also reported structural parameters, vibrational and electronic absorption spectra of polymer and substituted using quantum chemical methods. The geometrical parameters such as dipole moment, bond length and angles are reported at B3LYP/6-311++g** level of theory. In addition, the vibrational, electronic absorption spectra and NLO properties are also reported at the same level of theory. There is significant change in dipole moment and energy observed whereas symmetry, bond length and angles are resembling in Y-shaped and substituted polymer. The vibrational spectra of Y-shaped polymer (P1) having the intense peak is C-H stretching mode observed at $1258 \mathrm{~cm}-1$. These Theoretical vibrational modes are well matching with available experimental determinations. The method dependent and the along the X, Y and Z-direction hyperpolarizabilites also reported. This study confirms the polymer P1 and P2 showing first and second hyperpolarizability response whereas P3 do not show. The electronic absorption spectra for polymer and substituted polymers are also reported at the same level of theory using (TDDFT) approach. The wavelength of electronic transition, oscillator strength and HOMO-LUMO gap also reported.
\end{abstract}

\section{Hosted file}

IJQC.docx available at https://authorea.com/users/321356/articles/450645-nlo-properties-andspectroscopic-characterization-of-y-shaped-polymer-using-quantum-chemical-approach 